\title{
Diseases caused by the human parvovirus B19
}

Parvoviruses consist of single molecules of single stranded DNA surrounded by non-enveloped protein coats. They affect a wide range of animals (cats, dogs, rats, pigs, cattle, mink, rabbit, geese) and cause, in some of them, a variety of diseases (panleucopenia, cerebellar ataxia, enteritis, myocarditis, renal failure, fetal loss). B19 is the first human parvovirus. It was discovered in 1975 but has only been extensively investigated since 1980 when it was realised that it was the cause of appreciable human disease.

\section{Occurrence of B19 infection}

This is a common infection; about $70 \%$ of adults have serological evidence of past infection. Antibody is most commonly acquired between the ages of 5 and 15 years. The peak incidence of infections is in March, April, and May each year and epidemics tend to occur every three to five years. The infection is almost certainly worldwide as it has been found in every country (Europe, Scandinavia, America, Japan, Australia) where adequate studies have been done. Virus is infectious when given as nasal drops and the natural mode of transmission is almost certainly via the respiratory route from individuals incubating the infection. One week after exposure large amounts of virus are found in the blood for two or three days. At this time minor non-specific symptoms and any related to bone marrow failure (see below) occur. After about another week there is a second phase of illness characterised by a transient maculopapular rash or joint involvement, or both. At this stage there is a vigorous immune response, excretion of the virus has ceased, and the individual is immune for life.

\section{Rash illness due to B19 infection}

As with all common virus infections there is a wide range of disease. Wholly subclinical infections occur, more commonly in children than in adults. Infection with B19 may cause a wholly non-specific disease with a mild, transient fever and respiratory tract symptoms. The most common clinical symptom of infection is an erythematous rash to which a variety of diagnostic labels will be applied depending upon the nature of the rash and the context of its occurrence.

Erythema infectiosum or fifth disease (so called because it was the fifth of six childhood exanthems in an old classification) was first described about 100 years ago. There are usually no immediately prodromal symptoms but fever and mild respiratory tract symptoms may have occurred a week before. The rash begins with intense erythema of the cheeks (hence the synonym, slapped cheek disease). The edges of the red areas tend to be raised and there is relative circumoral pallor. The rash then appears on the arms and the legs as a discrete maculopapular erythema. The rash may become confluent in areas and then clears from the centre giving a lacy or reticular appearance especially over the lower limbs. The rash usually clears after a day or two but then has a tendency to recrudesce when the infected individual gets hot after exercise, bathing, or in sunlight.

Cases and outbreaks of erythema infectiosum throughout the world have been shown to be due to B19 infection. This particular diagnosis is usually made when there is a prominent slapped cheek component, or a lacy appearance to the rash or when an outbreak of such illness occurs in primary school children. The slapped cheek appearance does not always occur, however, and is particularly uncommon in adults. In such cases a diagnosis of rubella is often made and the other common clinical diagnoses in cases of B19 infection are allergic rash or unspecified viral rash. Lymphadenopathy is found more often in rubella but joint involvement is common in both infections (see below) and the seasonal incidence of the two is the same. Specific laboratory tests are the only way of distinguishing precisely between rubella and B19 infection.

The rash associated with B19 infection is not always erythematous. The occasional patient with recent B19 infection will present with easy bruising or purpura. Some have low platelet counts but most do not. The occasional case diagnosed clinically as Henoch-Schönlein purpura has been shown to be a recent $\mathrm{B} 19$ infection.

\section{Joint involvement in B19 infection}

Joint involvement has long been recognised as a complication of erythema infectiosum and with the advent of specific laboratory tests for B19 infection it is clear that this infection causes arthritis and arthralgia. In many respects it is equivalent to the arthropathy seen in rubella infections. It may occur 
with an erythematous rash or may be the sole presenting symptom. It occurs in only $5-10 \%$ of B19 infections of children but in $80 \%$ of infections in adult women. It usually involves the joints of the hands and wrists but may also involve knees and ankles. Arthralgia is the most common complaint but the joints are often stiff and swollen. In most instances the joint symptoms resolve within two weeks but in a small number of cases the symptoms may persist for months or years. Initially some patients have been classified as early rheumatoid arthritis but there is no evidence that B19 virus causes this disease.

\section{Haematological changes in B19 infection}

B19 virus infects the red blood cell precursors in the bone marrow and there is a cessation of erythropoiesis for about one week. This occurs in all persons infected with the virus but in haematologically normal people with haemoglobins of $110-140$ $\mathrm{g} / \mathrm{l}$ and red cells with a life span of 120 days there are no clinical effects, although the haemoglobin concentration temporarily drops by about $10 \mathrm{~g} / \mathrm{l}$. In those with chronic haemolytic anaemia, however, with an already low haemoglobin (steady state concentrations of 70-100 g/l) and red blood cells with a short life span (about 20 days) there is a temporary fall in haemoglobin to very low values and the patient is ill with the symptoms and signs of profound anaemia, which may require blood transfusion. This is called an aplastic crisis and is particularly a feature of sickle cell anaemia and hereditary spherocytosis. Over $95 \%$ of all cases of aplastic crisis that have been adequately investigated have been shown to be due to B19 virus infection and most occur in children under 15 years of age.

\section{B19 virus infection in pregnancy}

A transient but intense viraemia occurs as part of every B19 infection and should this occur during pregnancy there will be an opportunity for the virus to spread to the placenta and fetus. There is no doubt that B19 does cause fetal infection. It does not appear to cause birth defects like rubella virus infection but is a cause of loss of the pregnancy. If this occurs early in pregnancy it will be a spontaneous abortion and B19 infection appears to increase the likelihood of this threefold. If maternal B19 infection occurs in the second or third trimester it may be associated with hydrops fetalis due to a persistent fetal infection. This may result in late abortion or stillbirth. At present there is no need to alter the management of pregnancies complicated by B19 infection. The pregnancy may be lost but if it is not then a normal baby can be anticipated, although in many instances follow up has been for only a relatively short time.

\section{Diagnosis of $B 19$ infection}

The common rash, illness and joint symptoms occur at a time when virus can no longer be detected in serum or throat swabs in most cases. Diagnosis is therefore based on the detection of B19 specific IgM antibody that is present in serum for two to three months after infection. Past exposure (and therefore immunity) can be assessed by testing for specific antibody of the IgG class. This diagnostic approach is exactly that used for rubella and a clotted blood sample with full clinical details is all that is required to investigate suspicious rashes. Virus may still be present in the serum in cases of aplastic crises and should be looked for by immunological or molecular biological techniques. Detection of virus is the only satisfactory method of diagnosing B19 infection in hydropic fetuses.

Unfortunately there is still a severe shortage of reagents for B19 virus diagnostic tests and these are only available in a limited number of centres. Hopefully in the near future synthetic antigens will become available. Diagnostic antibody tests could then be applied on a much wider scale.

For further details the reader is referred to the following reviews and textbook descriptions.

\section{Further reading}

1 Anderson MJ. Parvoviruses as agents of human disease. In: Melnick JL, ed. Progress in medical virology. Basle: Karger, 1987;34:55-9.

2 Anderson MJ, Cherry JD. Parvovirus infection. In: Feigin D, Cherry JD, eds. Textbook of pediatric infectious diseases. 2nd ed. Philadelphia: WB Saunders, 1987:1646-53.

3 Anderson MJ, Pattison JR. Parvovirus. In: Weatherall DJ, Ledingham JGG, Warrell DA, eds. Oxford textbook of medicine. 2nd ed. Oxford: Oxford University Press, 1987:5.162-4.

4 Pattison JR. B19 virus-a pathogenic human parvovirus. Blood Reviews 1987;1:58-64.

5 Pattison JR. Parvoviruses and human disease. Boca Raton: CRC Press, 1988.

\author{
JR PatTison \\ Department of Medical Microbiology, \\ University College and Middlesex \\ School of Medicine, Rockefeller \\ Building, University Street, \\ London WC1E 6JJ
}

